\title{
Frequency tracking by method of least squares combined with channel estimation for OFDM over mobile wireless channels
}

\author{
Rainfield Y Yen ${ }^{1}$, Hong-Yu Liu ${ }^{2 *}$ and Chia-Sheng Tsai ${ }^{3}$
}

\begin{abstract}
To track frequency offset and time-varying channel in orthogonal frequency division multiplexing (OFDM) systems over mobile wireless channels, a common technique is, based on one OFDM training block sample, to apply the maximum-likelihood (ML) algorithm to perform joint frequency tracking and channel estimation employing some adaptive iteration processes. The major drawback of such joint estimation techniques is the local extrema problem arising from the highly nonlinear nature of the log-likelihood function. This makes the joint estimation process very difficult and complicated, and many a time the results are not very satisfactory if the algorithm is not well designed. In this study, rather than using the ML algorithm, we shall apply the method of least squares (LS) for frequency tracking utilizing repeated OFDM training blocks. As will be seen, by using such an LS approach, the frequency offset estimation requires no channel knowledge. The channel state can be estimated separately after the LS frequency offset correction. This not only circumvents the local extrema complication, but also obviates the need for the lengthy adaptive iteration process of joint estimation thus greatly simplifies the entire estimation process. Most importantly, our technique can achieve excellent estimation performance as compared to the usual ML algorithms.
\end{abstract}

Keywords: orthogonal frequency division multiplexing (OFDM), least squares (LS) estimation, maximum-likelihood (ML) estimation, carrier frequency synchronization, channel estimation.

\section{Introduction}

In orthogonal frequency division multiplexing (OFDM) communications over mobile wireless channels, after initial time and frequency acquisition, fine frequency offset and time variation of the channel state will continue to exist due to Doppler shift, instability of local oscillators, and multipath fading and hence must be continuously or frequently tracked [1]. To perform fine frequency tracking and channel estimation, the most widely adopted technique is the maximum-likelihood (ML) estimation algorithm. Usually, one OFDM symbol block is used as the training data. When using the ML technique to track frequency offset, one needs to have the channel knowledge. Likewise, to perform ML channel estimation, frequency offset estimation must be

\footnotetext{
* Correspondence: hongyu.liu@msa.hinet.net

${ }^{2}$ Department of Electrical Engineering, Fu Jen Catholic University, No. 510,

Zhongzheng Rd., Xinzhuang Dist., New Taipei City 24205, Taiwan

Full list of author information is available at the end of the article
}

given. Therefore, to track both fine frequency offset and the channel state, a popular method is to alternately estimate the frequency offset and channel state by adaptive iterations. In the literature, there exist many proposed ML algorithms of joint frequency tracking and channel estimation for both linear modulation and OFDM systems [2-6]. A major difficulty is the local extrema or multiple solution complication arising from the highly nonlinear nature of the multi-dimensional log-likelihood function. As a result, some schemes of simplification or approximation are often used to help solve the problem $[5,6]$. In addition, complex algorithms must usually be accompanied to aid the tedious search of the global solution [2-6]. How well such algorithms are designed will greatly affect the global solution convergence performance. When the initial frequency offset is large (close to half the subcarrier spacing), it usually becomes rather difficult to achieve global solution convergence. Carefully observing the simulation results of 
most of the cited works above, we can perceive that, when the initial frequency offset exceeds \pm 0.1 of the subcarrier spacing, the mean square errors (MSEs) or variances of both the frequency and channel estimators given in these works start to depart away from the Cramer-Rao bound (CRB) as signal-to-noise ratio (SNR) is increased. Thus, their tracking ranges are well below half or \pm 0.5 of the subcarrier spacing. This is due to the less accurate results arising from the approximations and/or simplifications when initial frequency offset values become large. In [1], by using the linear minimum-mean-square-error (LMMSE) combiner to optimally combine frequency offset estimators based on single time slot pilot samples from a received timedomain OFDM block, they successfully circumvent the multiple solution difficulty and meanwhile achieve very satisfactory joint estimation performance with wide tracking range (up to half the subcarrier spacing). Moreover, in [1], the estimator MSE will not depart away from the CRB at large SNRs. However, their algorithm still requires the lengthy adaptive iteration process due to joint estimations. Since, as stated earlier, it is necessary to continuously or frequently perform tracking of frequency and channel state, the lengthy adaptive iteration process, which is time-consuming, should be highly undesirable.

In this article, rather than using the ML technique, we apply the method of least squares (LS) to perform fine frequency tracking utilizing repeated OFDM training blocks. Our LS formulation for frequency offset estimation will not require channel knowledge. Then, based on the frequency offset estimator thus obtained, channel estimation is readily performed. This way, not only the multiple solution complication due to nonlinearity of the log-likelihood function can be avoided, but also the need for lengthy adaptive iterations is obviated. Thus, the entire process becomes much simpler and faster. Most important, our estimator performance outperforms those of the existing ML techniques. However, it is to be noted that the advantages and superiority of our technique are achieved at the expense of data transfer rate reduction due to the use of repeated training blocks.

The article is organized as follows: Section 2 presents the signal and system model. Section 3 delineates our LS formulation for frequency tracking. Section 4 addresses the channel estimation techniques after frequency offset correction. Section 5 analyzes our estimation performances. Then, Section 6 presents simulation results. Performance comparisons will be made between our LS algorithm and the usual ML algorithms based on single training block. Finally, Section 7 draws conclusions.

\section{Signal and system model}

Consider OFDM communications over mobile wireless channels. Assume time and frequency acquisitions have been completed. Due to Doppler shift, instability of the oscillators, and multipath fading, fine frequency offset and time variation of the channel state will continue to exist. Let $x_{n}, h_{n}$, and $w_{n}$, respectively, represent the discrete-time baseband data sample, channel impulse response (CIR), and noise sample at the $n$th time slot of a received time-domain OFDM block having the length of $N$ sample units or $T$ seconds. The fine frequency offset normalized to subcarrier spacing $\Delta f=1 / T$ is denoted as $\delta$. Then, discarding the cyclic prefix, the demodulated baseband received sample at the $n$th time slot can be expressed as

$$
r_{n}=e^{j 2 \pi n \delta / N} x_{n} * h_{n}+w_{n}=e^{j 2 \pi n \delta / N} \gamma_{n}+w_{n}, \quad n=0,1, \ldots, N-1,
$$

where * denotes convolution and $y_{n}=x_{n} * h_{n}$ is the received noise-free data sample which can be expressed in terms of frequency-domain quantities as

$$
y_{n}=x_{n} * h_{n}=\frac{1}{\sqrt{N}} \sum_{k=0}^{N-1} H_{k} X_{k} e^{j 2 \pi n k / N},
$$

where $H_{k}$ and $X_{k}$ are, respectively, the $k$ th subcarrier channel frequency response (CFR) and transmitted data symbol or the discrete Fourier transform (DFT) of $h_{n}$ and $x_{n}$. We have adopted the unitary DFT here for the signal data. Note that $\left\{w_{n}\right\}$ are independent, identically distributed (i.i.d.) complex Gaussian random variables (RVs) with zero mean and variance $\sigma_{w}^{2}$ and we shall use the familiar notation $w_{n} \sim N\left(0, \sigma_{w}^{2}\right)$ for convenience.

For frequency-selective channels, the discrete-time CFR is expressed as

$$
H_{k}=\sum_{m=0}^{\nu-1} h_{m} e^{-j 2 \pi m k / N}, \quad k=0,1, \ldots, N-1,
$$

where we have assumed the channel is causal and its length spans $v$ data samples. The discrete-time baseband CIRs $\left\{h_{m}\right\}$ are spatially uncorrelated. The DFT output at the receiver is given by

$$
\begin{gathered}
R_{k}=\frac{1}{\sqrt{N}} \sum_{n=0}^{N-1} r_{n} e^{-j 2 \pi n k / N}=\frac{1}{N} \sum_{n=0}^{N-1} e^{j 2 \pi n \delta / N}\left[\sum_{p=0}^{N-1} H_{p} X_{p} e^{j 2 \pi n p / N}\right] \\
e^{-j 2 \pi n k / N}+W_{k}, \quad k=0,1, \ldots, N-1, \\
\text { where } \quad W_{k}=\frac{1}{\sqrt{N}} \sum_{n=0}^{N-1} w_{n} e^{-j 2 \pi n k / N} \sim N\left(0, \sigma_{W}^{2}\right)=N\left(0, \sigma_{w}^{2}\right) .
\end{gathered}
$$

Apparently, $\left\{W_{k}\right\}$ are i.i.d. Gaussian RVs. With the completion of initial frequency acquisition, we can 
reasonably assume that the maximum fine frequency offset is less than half the subcarrier spacing or $\delta<0.5$ [1-6].

\section{Frequency tracking by the method of LS}

We shall use $P_{k}$ to denote training or pilot symbols to distinguish it from the data symbol $X_{k}$. To each transmitted block which is labeled as the initial block or 0th block, we append $L$ identical blocks (i.e., $N$ pilot symbols in each appended block are exact the same as those of the transmitted block). With this arrangement, the received sample of the $n$th time slot in the $l$ th block can be denoted as

$$
\tilde{r}_{l, n}=r_{n+l N}=\tilde{y}_{l, n}+\tilde{w}_{l, n,} \quad l=0,1, \ldots, \quad L, n=0,1, \ldots, N-1,
$$

where $\tilde{w}_{l, n}=w_{n+l N}$ is a complex Gaussian RV having zero mean and variance $\sigma_{w}^{2}$, and

$$
\begin{aligned}
\tilde{y}_{l, n} & =e^{j 2 \pi(n+l N) \delta / N} \frac{1}{\sqrt{N}} \sum_{k=0}^{N-1} H_{k} P_{k} e^{j 2 \pi(n+l N) k / N} \\
& =e^{j 2 \pi(n+l N) \delta / N} \frac{1}{\sqrt{N}} \sum_{k=0}^{N-1} H_{k} P_{k} e^{j 2 \pi n k / N} .
\end{aligned}
$$

In (6), we assume $L$ is not so large that the channel fading remains unchanged at least over $(L+1)$ blocks. Define the vectors $\tilde{\mathbf{r}}_{l}=\left[\tilde{r}_{l, 0}, \tilde{r}_{l, 1}, \ldots, \tilde{r}_{l, N-1}\right]^{T}$, $\tilde{\mathbf{y}}_{l}=\left[\tilde{y}_{l, 0}, \tilde{y}_{l, 1}, \ldots, \tilde{y}_{l, N-1}\right]^{T}$, and $\tilde{\mathbf{w}}_{l}=\left[\tilde{w}_{l, 0}, \tilde{w}_{l, 1}, \ldots, \tilde{w}_{l, N-1}\right]^{T}$ with $T$ denoting transpose. Using (6), we can rewrite (5) in vector form as

$$
\begin{aligned}
& \tilde{\mathbf{r}}_{l}=\tilde{\mathbf{y}}_{l}+\tilde{\mathbf{w}}_{l}=\tilde{\mathbf{y}}_{l-1} e^{j 2 \pi \delta}+\tilde{\mathbf{w}}_{l} \\
& =\left(\tilde{\mathbf{r}}_{l-1}-\tilde{\mathbf{w}}_{l-1}\right) e^{j 2 \pi \delta}+\tilde{\mathbf{w}}_{l} \\
& =\tilde{\mathbf{r}}_{l-1} e^{j 2 \pi \delta}-\tilde{\mathbf{w}}_{l-1} e^{j 2 \pi \delta}+\tilde{\mathbf{w}}_{l}, \quad l=1,2, \ldots, L .
\end{aligned}
$$

It is easy to see that the combined noise vector $\mathbf{e}_{l}=-\tilde{\mathbf{w}}_{l-1} e^{j 2 \pi \delta}+\tilde{\mathbf{w}}_{l}$ is a complex Gaussian random vector with mean $\mathbf{0}$ and variance $2 \sigma_{w}^{2} \mathbf{I}$. We can stack the vectors of (7) to obtain

$$
\left[\begin{array}{c}
\tilde{\mathbf{r}}_{1} \\
\tilde{\mathbf{r}}_{2} \\
\vdots \\
\tilde{\mathbf{r}}_{L}
\end{array}\right]=\left[\begin{array}{c}
\tilde{\mathbf{r}}_{0} \\
\tilde{\mathbf{r}}_{1} \\
\vdots \\
\tilde{\mathbf{r}}_{L-1}
\end{array}\right] e^{j 2 \pi \delta}+\left[\begin{array}{c}
\mathbf{e}_{1} \\
\mathbf{e}_{2} \\
\vdots \\
\mathbf{e}_{L}
\end{array}\right] .
$$

We further define three $N(L+1) \times 1$ vectors $\mathbf{r}_{B}=\left[\tilde{\mathbf{r}}_{0}^{T}, \tilde{\mathbf{r}}_{1}^{T}, \ldots, \tilde{\mathbf{r}}_{L-1}^{T}\right]^{T}, \quad \mathbf{r}_{B}=\left[\tilde{\mathbf{r}}_{0}^{T}, \tilde{\mathbf{r}}_{1}^{T}, \ldots, \tilde{\mathbf{r}}_{L-1}^{T}\right]^{T}, \quad$ and $\mathbf{e}=\left[\mathbf{e}_{1}^{T}, \mathbf{e}_{2}^{T}, \ldots, \mathbf{e}_{L}^{T}\right]^{T}$. Equation (8) can then be rewritten as

$$
\mathbf{r}_{B} e^{j 2 \pi \delta}=\mathbf{r}_{A}-\mathbf{e}
$$

We can view (9) as an over-determined system of complex linear simultaneous equations in one complex variable $x=e^{j 2 \pi \delta}$. Then, we can apply the method of LS to obtain the estimate of $x$ as

$$
\hat{x}=\left(\mathbf{r}_{B}^{H} \mathbf{r}_{B}\right)^{-1} \mathbf{r}_{B}^{H} \mathbf{r}_{A}=\frac{\tilde{\mathbf{r}}_{0}^{H} \tilde{\mathbf{r}}_{1}+\tilde{\mathbf{r}}_{1}^{H} \tilde{\mathbf{r}}_{2}+\cdots+\tilde{\mathbf{r}}_{L-1}^{H} \tilde{\mathbf{r}}_{L}}{\left\|\mathbf{r}_{B}\right\|^{2}}=\frac{1}{\left\|\mathbf{r}_{B}\right\|^{2}} \sum_{l=1}^{L} \tilde{\mathbf{r}}_{l-1}^{H} \tilde{\mathbf{r}}_{l,}
$$

where $H$ denotes Hermitian transpose. Then, an estimate of $\delta$ can be found as

$$
\hat{\delta}=\frac{1}{2 \pi} \tan ^{-1}\left[\frac{\operatorname{Im}\left(\sum_{l=1}^{L} \tilde{\mathbf{r}}_{l-1}^{H} \tilde{\mathbf{r}}_{l}\right)}{\operatorname{Re}\left(\sum_{l=1}^{L} \tilde{\mathbf{r}}_{l-1}^{H} \tilde{\mathbf{r}}_{l}\right)}\right],
$$

where $\operatorname{Re}(\cdot)$ and $\operatorname{Im}(\cdot)$, respectively, denote the real and imaginary parts. It is apparent that the range of $\hat{\delta}$ given by (11) is \pm 0.5 or half the subcarrier spacing, and it has no multiple solution problem, requires no channel knowledge and only takes a one step computation process without adaptive iterations. However, these advantages are not without cost. The price paid is that the estimator of (11) requires the use of more than one OFDM training blocks. This results in a reduction of system throughput or data transfer rate.

It is to be noted that (11) is based on the premise that fading remains unchanged over at least $L+1$ OFDM blocks. In other words, we have assumed slow quasi-static fading. This implies that the maximum Doppler frequency must satisfy $f_{M}=v f_{c} / c \ll 1 / T$ corresponding to a mobile speed $v \ll c /\left(f_{c} T\right)$, where $c$ is the speed of light, $T$ is one OFDM block length in seconds, and $f_{c}$ is the carrier frequency in Hz. Taking an 802.11a standard with $f_{c}=5 \mathrm{GHz}$ and $\Delta f=1 / T=312.5 \mathrm{kHz}$, this requires $v \ll 67,500 \mathrm{~km} / \mathrm{h}$. Apparently, this requirement for slow quasi-static fading is easily met in practice. For example, if $v=60 \mathrm{~km} / \mathrm{h}$, then $f_{M} \approx 10^{-3} / T$ which is one thousandth of an OFDM block, it is thus reasonable to assume fading to remain unchanged over several hundred OFDM blocks.

In many applications, channel may have fast time variations. Then, our algorithm may lose its advantage, viz., fast processing due to no need for iterations. In such cases, the LMMSE algorithm of [1], which is shown better than most other ML schemes in the literature, may be the best choice. However, if time variation is not so fast or is only moderately fast (in between fast and slow varying), our LS algorithm can be still quite useful as we can cope with the moderately fast time-varying environment by reducing the number of repeated blocks. 


\section{Channel estimation}

After the fine frequency offset has been corrected, the channel estimation can readily be performed. Here, both the methods of LS and the ML technique will be investigated for channel estimation.

\subsection{LS channel estimation}

Given $\hat{\delta}$, we can correct the offset by multiplying (5) by $e^{-j 2 \pi(n+l N) \hat{\delta} / N}$ to obtain $r_{l, n}^{\prime}=\tilde{r}_{l, n} e^{-j 2 \pi(n+l N) \hat{\delta} / N}$ (before $\tilde{r}_{l, n}$ is fed to the DFT). If the estimate is of good accuracy, the estimate error $\Delta \delta=\delta-\hat{\delta}$ will be very small. Then, after frequency offset correction, the $k$ th subcarrier DFT output (during training mode) can be approximated as

$$
\begin{aligned}
& R_{l, k}^{\prime}=\frac{1}{\sqrt{N}} e^{j 2 \pi l \Delta \delta} \sum_{n=0}^{N-1} e^{j 2 \pi n \Delta \delta / N} \\
& {\left[\frac{1}{\sqrt{N}} \sum_{p=0}^{N-1} H_{p} P_{p} e^{j 2 \pi n p / N}\right] e^{-j 2 \pi n k / N}+W_{l, k}^{\prime}} \\
& =\frac{e^{j 2 \pi l \Delta \delta}}{N} H_{k} P_{k} \sum_{n=0}^{N-1} e^{j 2 \pi n \Delta \delta / N} \\
& +\frac{e^{j 2 \pi l \Delta \delta}}{N} \sum_{p=0}^{N-1} H_{p} P_{p} \sum_{n=0}^{N-1} e^{j 2 \pi(p-k+\Delta \delta) n / N}+W_{l, k}^{\prime} \\
& \quad p \neq k \\
& \approx H_{k} P_{k} e^{j \pi(2 l N+N-1) \Delta \delta / N}+W_{l, k}^{\prime} \quad l=0,1, \ldots, L, \\
& \approx H_{k} P_{k}\left(1+j \alpha_{l} \Delta \delta\right)+W_{l, k}^{\prime} \quad l=0,1,10
\end{aligned}
$$

where $\alpha_{l} \equiv \pi(2 l N+N-1) / N$, $W_{l, k}^{\prime}=\sum_{n=0}^{N-1} \tilde{w}_{l, n} e^{-j 2 \pi(n+l N) \hat{\delta} / N} e^{-j 2 \pi n k / N} \sim N\left(0, \sigma_{W}^{2}\right), \quad$ and we have used the approximations $e^{j \alpha_{l} \Delta \delta} \approx 1+j \alpha_{l} \Delta \delta$, $\sum_{n=0}^{N-1} e^{j 2 \pi n \Delta \delta / N}=\frac{e^{j \pi(N-1) \Delta \delta / N} \sin [\pi \Delta \delta]}{\sin [\pi \Delta \delta / N]} \approx N e^{j \pi(N-1) \Delta \delta / N}$, and $p-k+\Delta \delta \approx p-k$.

If the frequency offset correction were perfect (offsetfree), then $\Delta \delta=0$ and (12) would reduce to

$$
\bar{R}_{l, k}^{\prime}=H_{k} P_{k}+W_{l, k}^{\prime} \quad l=0,1, \ldots, L,
$$

where $\bar{R}_{l, k}^{\prime}$ stands for the offset-free DFT output. There are $L+1$ equations in (13) and they form a system of simultaneous linear equations for one variable $H_{k}$ and we can thus easily apply the method of LS to get the channel estimators as

$$
\hat{H}_{k}=\left(\mathbf{P}_{k}^{H} \mathbf{P}_{k}\right)^{-1} \mathbf{P}_{k}^{H} \overline{\mathbf{R}}_{k}^{\prime}=\frac{\sum_{l=0}^{L} \bar{R}_{l, k}^{\prime}}{(L+1) P_{k}} \quad k=0,1, \ldots, N-1,
$$

where $\mathbf{P}_{k}=P_{k}[1,1, \ldots, 1]^{T}$ is an $(L+1) \times 1$ vector and $\overline{\mathbf{R}}_{k}^{\prime}=\left[\bar{R}_{0, k^{\prime}}^{\prime} \bar{R}_{1, k}^{\prime}, \ldots, \bar{R}_{L, k}^{\prime}\right]^{T}$.

In reality, perfect frequency offset correction is not very likely to happen. That is, it is not very likely to obtain the offset-free outputs $\left\{\bar{R}_{l, k}^{\prime}\right\}$ of (13). The best one can do is to use the $\left\{R_{l, k}^{\prime}\right\}$ of (12). Thus, in practice, we shall take

$$
\hat{H}_{k}=\frac{\sum_{l=0}^{L} R_{l, k}^{\prime}}{(L+1) P_{k}}, \quad k=0,1, \ldots, N-1,
$$

Note that only one sample (the $k$ th sample $R_{l, k}^{\prime}$ ) in each training block (frequency-domain OFDM block) has been selected to estimate $H_{k}$ and there are $L+1$ blocks resulting in only $L+1$ samples used for the LS channel estimation. In order not to reduce data transfer rate too much, $L$ is not to be too large. Thus, with the small number of $L+1$ samples used, the LS estimator $\hat{H}_{k}$ is expected to perform not quite so satisfactorily, though perfectly workable. We will thus seek an alternative approach using the ML technique to be described next.

\section{2. ML channel estimation}

To perform ML channel estimation, we first need to find the log-likelihood function in terms of the variables to be estimated. These variables must be independent of each other. We shall assume a frequency-selective Rayleigh fading channel of dispersion length $v$ having the CIR vector $\mathbf{h}=\left[h_{0}, h_{1}, \ldots, h_{v-1}\right]^{T}$. Then $\left\{h_{m}, m=0,1, \ldots \nu\right\}$ are uncorrelated and hence independent of each other. However, the CFRs $\left\{H_{k}, k=0,1, \ldots, N\right\}$, which are DFTs of $\left\{h_{m}\right\}$, are obviously correlated and hence dependent upon each other. Consequently, unlike the above case of LS channel estimation where CFR can be estimated directly due to the special one variable over-determined system formulation, here we must first obtain the ML CIR estimator $\hat{\mathbf{h}}$ from a log-likelihood function in terms of $\mathbf{h}$ from which we then get the CFR estimator $\hat{\mathbf{H}}$ through DFT.

Now, assume that frequency offset has been corrected so that $\delta=0$. Using (5) and (6) with $\delta=0$, we can readily get

$$
\tilde{\mathbf{r}}_{l}=\mathbf{y}_{0}+\hat{\mathbf{w}}_{l}=\frac{1}{\sqrt{N}} \mathbf{F}_{N}^{H} \mathbf{P} F_{v} \mathbf{h}+\hat{\mathbf{w}}_{l},
$$


where $\mathbf{y}_{0}=\frac{1}{\sqrt{N}} \mathbf{F}_{N}^{H} \mathbf{P F}_{v} \mathbf{h}$ is the offset-free noiseless received signal vector with $\mathbf{P}=\operatorname{diag}\left\{P_{0}, P_{1}, \ldots, P_{N-1}\right\}$ being a diagonal matrix and

$$
\mathbf{F}_{v}=\left[\begin{array}{cccc}
1 & 1 & \cdots & 1 \\
1 & e^{-j 2 \pi / N} & \cdots & e^{-j 2 \pi(v-1) / N} \\
\vdots & \vdots & \cdots & \vdots \\
1 & e^{-j 2 \pi(N-1) / N} & \cdots & e^{-j 2 \pi(N-1)(v-1) / N}
\end{array}\right] .
$$

Then, stacking $L+1$ repeated blocks, we have

$$
\left[\begin{array}{c}
\tilde{\mathbf{r}}_{0} \\
\tilde{\mathbf{r}}_{1} \\
\vdots \\
\tilde{\mathbf{r}}_{L-1}
\end{array}\right]=\mathbf{r}_{B}=\left[\begin{array}{c}
\mathbf{y}_{0} \\
\mathbf{y}_{0} \\
\vdots \\
\mathbf{y}_{0}
\end{array}\right]+\left[\begin{array}{c}
\mathbf{w}_{0} \\
\mathbf{w}_{1} \\
\vdots \\
\mathbf{w}_{L-1}
\end{array}\right] .
$$

The log-likelihood function for (18) is given by

$$
\Lambda=-N \ln \pi \sigma_{w}^{2}-\frac{1}{\sigma_{w}^{2}} \sum_{l=0}^{L}\left\|\tilde{\mathbf{r}}_{l}-\frac{1}{\sqrt{N}} \mathbf{F}_{N}^{H} \mathbf{P F}_{v} \mathbf{h}\right\|^{2} .
$$

From (19), it can readily be shown that the ML estimate of $\mathbf{h}$ is given by

$$
\hat{\mathbf{h}}=\frac{1}{\sqrt{N}}\left(\mathbf{F}_{v}^{H} \mathbf{P}^{H} \mathbf{P} \mathbf{F}_{v}\right)^{-1} \mathbf{F}_{v}^{H} \mathbf{P}^{H} \mathbf{F}_{N}\left(\frac{1}{L+1} \sum_{l=0}^{L} \tilde{\mathbf{r}}_{l}\right),
$$

Training sequence with constant amplitude has been proven optimal for channel estimation [7]. Chu sequence [8], for example, falls onto this category. Using a Chu sequence $\left\{P_{k}=e^{j \pi m k^{2} / N}, m\right.$ being any integer relatively prime to $N\}$ results in $\mathbf{P}^{H} \mathbf{P}=\mathbf{I}_{N}$. Then, (20) can be simplified to

$$
\hat{\mathbf{h}}=\frac{1}{N \sqrt{N}} \mathbf{F}_{v}^{H} \mathbf{P}^{H} \mathbf{F}_{N}\left(\frac{1}{L+1} \sum_{l=0}^{L} \tilde{\mathbf{r}}_{l}\right),
$$

where the fact $\mathbf{F}_{v}^{H} \mathbf{F}_{v}=N \mathbf{I}_{v}$ has been used. Note that, by using a Chu sequence, (21) can avoid the computation of matrix inversion given in (20). The estimate of the CFR vector $\mathbf{H}=\left[H_{0}, H_{1}, \ldots, H_{N-1}\right]^{T}$ can be readily obtained as

$$
\hat{\mathbf{H}}=\mathbf{F}_{v} \hat{\mathbf{h}} \text {. }
$$

Since all samples in each of the entire OFDM block are utilized in this ML estimation, we have used a total of $N(L+1)$ samples for this ML channel estimation. As a result, we expect that the ML channel estimator will outperform the previous LS channel estimator. Later in simulations, we shall make performance comparisons between the CFR estimators given by (15) and (22).

\section{Analysis of estimation performance}

\subsection{Frequency tracking performance}

From (11), we see that $\sum_{l=1}^{L} \tilde{\mathbf{r}}_{l-1}^{H} \tilde{\mathbf{r}}_{l}=\left|\sum_{l=1}^{L} \tilde{\mathbf{r}}_{l-1}^{H} \tilde{\mathbf{r}}_{l}\right| e^{j 2 \pi \hat{\delta}}$.

Then, using the fact that $\tilde{\mathbf{y}}_{l}=\tilde{\mathbf{y}}_{0} e^{j 2 \pi l \delta}$, we can readily show that, for high SNR and small estimate error $\hat{\delta}-\delta \approx \frac{1}{2 \pi L|| \tilde{\mathbf{y}}_{0} \|^{2}} \operatorname{Im}\left[\tilde{\mathbf{y}}_{0}^{H} \tilde{\mathbf{w}}_{L} e^{-j 2 \pi L \delta}+\tilde{\mathbf{w}}_{0}^{H} \tilde{\mathbf{y}}_{0}\right]$.

$$
\hat{\delta}-\delta \approx \frac{1}{2 \pi L|| \tilde{\mathbf{y}}_{0} \|^{2}} \operatorname{Im}\left[\tilde{\mathbf{y}}_{0}^{H} \tilde{\mathbf{w}}_{L} e^{-j 2 \pi L \delta}+\tilde{\mathbf{w}}_{0}^{H} \tilde{\mathbf{y}}_{0}\right] .
$$

Note that $\tilde{\mathbf{y}}_{0}=\left[\tilde{y}_{0,0}, \tilde{y}_{0,1}, \ldots, \tilde{y}_{0, N-1}\right]^{T}$ is the noiseless received signal vector of the initial training block, with $\tilde{\gamma}_{0, n}=e^{j 2 \pi n \delta / N} \frac{1}{\sqrt{N}} \sum_{k=0}^{N-1} H_{k} P_{k} e^{j 2 \pi n k / N}, n=0,1, \ldots, N-1$ (see Equation (6)). For given $\tilde{\mathbf{y}}_{0}$ and $\delta$, we easily see that the mean $E[\Delta \delta]=E[\hat{\delta}-\delta]=0$. Hence, for small errors at high SNR, the frequency offset estimate is unbiased. Using (23), the variance or MSE of the estimate can be evaluated as

$$
E\left[(\hat{\delta}-\delta)^{2}\right]=\frac{\sigma_{w}^{2}}{4 \pi^{2} L^{2}\left\|\tilde{\mathbf{y}}_{0}\right\|^{2}}=\frac{1}{4 \pi^{2} L^{2} N \gamma} .
$$

Note that the block signal power $\left\|\tilde{y}_{0}\right\|^{2}$ will increase with $N$. We have thus defined the block SNR as $\gamma=\left\|\tilde{\mathbf{y}}_{0}\right\|^{2} / N \sigma_{w}^{2}$. We can see that for a given $\gamma$, as $L$ and/or $N$ are increased, the estimate gets better.

\subsection{Channel estimation performance}

\subsubsection{Estimation by method of $L S$}

We substitute (12) into (15) to get

$$
\hat{H}_{k}=H_{k}+\frac{H_{k} \sum_{l=0}^{L}\left[j \alpha_{l} \Delta \delta\right]}{(L+1)}+\frac{1}{(L+1) P_{k}} \sum_{l=0}^{L} W_{l, k^{\prime}}^{\prime}
$$

whence, for given $H_{k}$ and $\delta$, the mean of $\hat{H}_{k}$ is

$$
E\left[\hat{H}_{k}\right]=H_{k}
$$

where we have used $E[\Delta \delta]=0$. Hence, for small offset errors at high SNR, the channel estimate is unbiased. Then, using (23), (24), and (25), we can readily calculate the channel estimator variance or MSE (at given $H_{k}$ and $\delta)$ as

$$
E\left[\left|\hat{H}_{k}-H_{k}\right|^{2}\right]=\frac{C\left|H_{k}\right|^{2} \sigma_{W}^{2}}{\left\|\mathbf{y}_{0}\right\|^{2}}+\frac{\sigma_{W}^{2}}{(L+1)\left|P_{k}\right|^{2}},
$$


where

$$
C=\frac{(N L+N-1)^{2}}{4 N^{3} L^{2}} .
$$

The overall average variance is defined as

$$
\frac{1}{N} E\left[\|\Delta \mathbf{H}\|^{2}\right]=\frac{1}{N} \sum_{k=0}^{N-1} E\left[\left|\hat{H}_{k}-H_{k}\right|^{2}\right] .
$$

Equations (26) to (29) give the theoretical mean and variance of the ML channel estimators when the frequency offset estimate is imperfect. From (27), we see that, with a given training sequence $\left\{P_{k}\right\}$, the channel estimate variance is a function of $H_{k}$. If frequency offset estimate were perfect and a Chu sequence had been used, we would get

$$
\frac{1}{N} E\left[\|\Delta \mathbf{H}\|^{2}\right]=E\left[\left|\hat{H}_{k}-H_{k}\right|^{2}\right]=\frac{\sigma_{W}^{2}}{L+1} .
$$

\subsubsection{Estimation by $M L$ technique}

With imperfect frequency offset estimate as most likely would be the usual case, we would actually have

$$
\tilde{\mathbf{r}}_{l}=\mathbf{D}_{l, \Delta \delta} \mathbf{y}_{0}+\hat{\mathbf{w}}_{l}=\frac{1}{\sqrt{N}} \mathbf{D}_{l, \Delta \delta} \mathbf{F}_{N}^{H} \mathbf{P} \mathbf{F}_{v} \mathbf{h}+\hat{\mathbf{w}}_{l},
$$

where $\Delta \delta=\delta-\hat{\delta}$ is the frequency offset estimate error which is very small and

$$
\begin{aligned}
\mathbf{D}_{l, \Delta \delta}=\operatorname{diag}\left\{e^{j 2 \pi(0+l N) \Delta \delta / N}, e^{j 2 \pi(1+l N) \Delta \delta / N}, \ldots, e^{j 2 \pi[(N-1)+l N] \Delta \delta / N}\right\} \\
\approx \operatorname{diag}\{1+j 2 \pi(0+l N) \Delta \delta / N, 1+j 2 \pi(1+l N) \Delta \delta / N, \cdots, 1 \\
\quad+j 2 \pi[(N-1)+l N] \Delta \delta / N\} \\
=\mathbf{I}_{N}+\frac{j 2 \pi \Delta \delta}{N} \operatorname{diag}\{0+l N, 1+l N, \cdots,(N-1)+l N\} \\
=\mathbf{I}_{N}+\frac{j 2 \pi \Delta \delta}{N}\left(\mathbf{D}_{N}+l N \mathbf{I}_{N}\right),
\end{aligned}
$$

and

$$
\mathbf{D}_{N}=\operatorname{diag}\{0,1, \cdots, N-1\} .
$$

Substituting (31) into (20), we can readily get

$$
\begin{aligned}
\widehat{\mathbf{h}}= & \mathbf{h}+\left(\mathbf{F}_{v}^{H} \mathbf{P}^{H} \mathbf{P} \mathbf{F}_{v}\right)^{-1} \mathbf{F}_{v}^{H} \mathbf{P}^{H} \mathbf{F}_{N} \\
& \left(\sum_{l=0}^{L}\left[\frac{j 2 \pi \Delta \delta}{N^{2}(L+1)}\left(\mathbf{D}_{N}+l N \mathbf{I}_{N}\right) \mathbf{F}_{N}^{H} \mathbf{P} \mathbf{F}_{v} \mathbf{h}+\frac{1}{\sqrt{N}(L+1)} \widetilde{\mathbf{w}}_{l}\right]\right) \\
= & \mathbf{h}+\frac{j 2 \pi \Delta \delta}{N^{2}}\left(\mathbf{F}_{v}^{H} \mathbf{P}^{H} \mathbf{P} \mathbf{F}_{v}\right)^{-1} \mathbf{F}_{v}^{H} \mathbf{P}^{H} \mathbf{F}_{N}\left(\mathbf{D}_{N}+\frac{L N}{2} \mathbf{I}_{N}\right) \mathbf{F}_{N}^{H} \mathbf{P} \mathbf{F}_{v} \mathbf{h} \\
+ & \frac{1}{\sqrt{N}(L+1)}\left(\mathbf{F}_{v}^{H} \mathbf{P}^{H} \mathbf{P} \mathbf{F}_{v}\right)^{-1} \mathbf{F}_{v}^{H} \mathbf{P} \mathbf{F}_{N} \sum_{l=0}^{L} \widetilde{\mathbf{w}}_{l} .
\end{aligned}
$$

With $E[\Delta \delta]=0$, we can readily see from (34) that

$$
E[\hat{\mathbf{h}}]=\mathbf{h} .
$$

Therefore, the estimator $\hat{\mathbf{h}}$ is also unbiased.

Defining $\Delta \mathbf{h}=\hat{\mathbf{h}}-\mathbf{h}$, we can compute the covariance as follows:

$$
\begin{aligned}
& \operatorname{Cov}[\Delta \mathbf{h}]=E\left[\Delta \mathbf{h} \cdot \Delta \mathbf{h}^{H}\right] \\
& =\frac{4 \pi^{2} E\left[\Delta \delta^{2}\right]}{N^{4}}\left(\mathbf{F}_{v}^{H} \mathbf{P}^{H} \mathbf{P} \mathbf{F}_{v}\right)^{-1} \mathbf{F}_{v}^{H} \mathbf{P}^{H} \mathbf{F}_{N}\left(\mathbf{D}_{N}+\frac{L N}{2} \mathbf{I}_{N}\right) \\
& \mathbf{F}_{N}^{H} \mathbf{P} \mathbf{F}_{v} \mathbf{h} \mathbf{h}^{H} \mathbf{F}_{v}^{H} P^{H} \mathbf{F}_{N}\left(\mathbf{D}_{N}+\frac{L N}{2} \mathbf{I}_{N}\right) \mathbf{F}_{N}^{H} \mathbf{P} \mathbf{F}_{v}\left[\left(\mathbf{F}_{v}^{H} \mathbf{P}^{H} \mathbf{P} \mathbf{F}_{v}\right)^{-1}\right]^{H} \\
& +\frac{\sigma_{w}^{2}}{L+1}\left(\mathbf{F}_{v}^{H} \mathbf{P}^{H} \mathbf{P} \mathbf{F}_{v}\right)^{-1} \mathbf{F}_{v}^{H} \mathbf{P} \mathbf{P}^{H} \mathbf{F}_{v}\left[\left(\mathbf{F}_{v}^{H} \mathbf{P}^{H} \mathbf{P} \mathbf{F}_{v}\right)^{-1}\right]^{H}
\end{aligned}
$$

Now using a Chu sequence such that $\mathbf{P}^{H} \mathbf{P}=\mathbf{I}_{N}$, (36) reduces to

$$
\begin{aligned}
& \operatorname{Cov}[\Delta \mathbf{h}]=E\left[\Delta \mathbf{h} \cdot \Delta \mathbf{h}^{H}\right] \\
= & \frac{4 \pi^{2} E\left[\Delta \delta^{2}\right]}{N^{6}} \mathbf{F}_{v}^{H} \mathbf{P}^{H} \mathbf{F}_{N}\left(\mathbf{D}_{N}+\frac{L N}{2} \mathbf{I}_{N}\right) \mathbf{F}_{N}^{H} \mathbf{P} \mathbf{F}_{v} \mathbf{h} \mathbf{h}^{H} \mathbf{F}_{v}^{H} \mathbf{P}^{H} \mathbf{F}_{N} \\
& \left(\mathbf{D}_{N}+\frac{L N}{2} \mathbf{I}_{N}\right) \mathbf{F}_{N}^{H} \mathbf{P} \mathbf{F}_{v}+\frac{\sigma_{w}^{2}}{N(L+1)} \mathbf{I}_{v} \\
= & \frac{\sigma_{w}^{2}}{N^{5} L^{2}\left\|\mathbf{y}_{0}\right\|^{2}} \mathbf{F}_{v}^{H} \mathbf{P}^{H} \mathbf{F}_{N}\left(\mathbf{D}_{N}+\frac{L N}{2} \mathbf{I}_{N}\right) \mathbf{y}_{0} \mathbf{y}_{0}^{H} \\
& \left(\mathbf{D}_{N}+\frac{L N}{2} \mathbf{I}_{N}\right) \mathbf{F}_{N}^{H} \mathbf{P} \mathbf{F}_{v}+\frac{\sigma_{w}^{2}}{N(L+1)} \mathbf{I}_{v \prime}
\end{aligned}
$$

where $\mathbf{y}_{0}$ has been defined earlier in Section 4 and $E\left[\Delta \delta^{2}\right]=\frac{\sigma_{w}^{2}}{4 \pi^{2} L^{2}\left\|\tilde{\mathbf{y}}_{0}\right\|^{2}}=\frac{\sigma_{w}^{2}}{4 \pi^{2} L^{2}\left\|\mathbf{y}_{0}\right\|^{2}}$ as from

Note that if the estimate of frequency offset were perfect, i.e., $E\left[\Delta \delta^{2}\right]=0$, we would have

$$
\operatorname{Cov}[\Delta \mathbf{h}]=\frac{\sigma_{w}^{2}}{N(L+1)} \mathbf{I},
$$

and hence

$$
E\left[|\Delta \mathbf{h}|^{2}\right]=\frac{v \sigma_{w}^{2}}{N(L+1)}
$$

This leads to

$$
\frac{1}{N} E\left[|\Delta \mathbf{H}|^{2}\right]=E\left[|\Delta \mathbf{h}|^{2}\right]=\frac{v \sigma_{w}^{2}}{N(L+1)}=\frac{v \sigma_{W}^{2}}{N(L+1)} .
$$

Comparing (30) and (40), it is apparent that the ML channel estimation outperforms the LS channel estimation. 


\section{Simulation results}

In this section, we shall present various performance results of our LS frequency tracking combined with channel estimation in OFDM systems. Also included will be the QAM symbol error rate (SER) performance of an OFDM system employing our tracking algorithm in frequency-selective Rayleigh fading channels. Comparison will be made with the usual ML techniques based on one OFDM training block.

For a frequency-selective channel of dispersion length $v=9$, we choose a given exponential power profile with normalized channel power, i.e., $\sum_{m=0}^{8}\left|h_{m}\right|^{2}=1$, Figure 1 gives the variance or MSE of the frequency offset estimator $E\left[(\hat{\delta}-\delta)^{2}\right]$ as a function of SNR $\gamma$ for three values of $L=1,3,9$ as obtained from Monte Carlo simulations as well as from the theoretical result of (24). We have used 64-point DFT $(N=64)$ and assume an actual frequency offset of $\delta=0.2$. From the figure, we see that the simulated and theoretical curves coincide well at high SNR values as expected since the theoretical result of (24) was derived based on high SNR values. Also incorporated in Figure 1 for comparison purpose is the CRB for the usual ML frequency estimator based on one OFDM block as the training sample as for [1] and

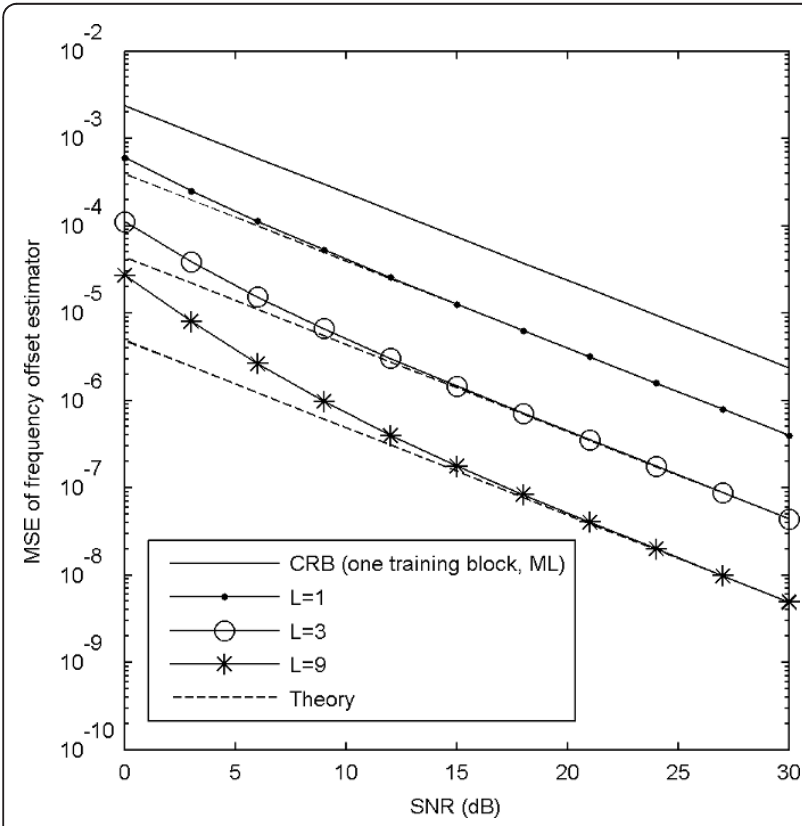

Figure 1 Performance of frequency tracking by method of least squares using $L+1$ OFDM training blocks. With initial frequency offset $\delta=0.2$ and for $L=1,3,9$, shown here are MSE of frequency offset estimator versus SNR curves by Monte Carlo simulations as well as by theory. The CRB curve is for ML frequency estimator using one OFDM training block. other earlier cited ML algorithms employing adaptive iterations for joint frequency tracking and channel estimation. After LS frequency tracking, using the LS channel estimation for the same frequency-selective channel with the same offset $\delta=0.2$, Figure 2 gives the CFR estimator variance or MSE against SNR obtained from Monte Carlo simulations as well as from the theoretical results of (29) under imperfect frequency offset estimations for the same three $L$ values. Also incorporated in Figure 2 for comparison is the CRB for the usual ML CFR estimator based on one OFDM block as the training sample. We see that this CRB lies in between the $L$ $=3$ and the $L=9$ LS curves. Thus, roughly, we may need to use at least $L=6$ to obtain better LS channel estimators when compared with the usual one-blocksample-based ML channel estimator. Then, Figure 3 presents the MSE versus SNR curves for the multipleblock-sample-based ML CFR estimator as obtained from Monte Carlo simulations as well as from the theoretical results of (37) under imperfect frequency tracking for $L$ $=1,3,9$. This time, we see that the multiple-block-sample-based ML channel estimators have MSEs lower than the CRB for the one-block-sample-based ML channel estimator as it should. Apparently, the ML channel estimator outperforms the LS channel estimator as predicted earlier by theory.

For the same frequency-selective channel system as for Figures 1, 2, and 3, the same performance curves are plotted in Figures 4, 5, and 6 for the case of $\delta=0.48$. As expected, with the larger initial frequency offset of 0.48 , estimator performances are degraded. The degradations are especially pronounced at low SNR values as can easily be observed from the figures.

It is important to note that comparison of different algorithms may not be always fair due to different system conditions applied to different algorithms. Thus, making trade-off decisions between different algorithms may well be intuitional. Many a time, the intuitional decision is quite obvious and hence rather easy to make. In our cases here, it is certainly unfair to compare the CRB for an ML estimator based on one training block sample with the estimator MSE using multiple training block samples. Nonetheless, the key point here is, by avoiding adaptive iterations, we can achieve considerable time-saving and substantial process simplification, meanwhile accomplish the purpose of lower estimator MSE. In fact, one can also go to great lengths to apply the ML algorithm to perform the usual joint frequency tracking and channel estimation but using multiple training block samples. This will bring the CRBs down a little further than our estimator MSEs. But, such processes would be overly complex and much time-consuming, and hence prohibitively expensive to implement. 


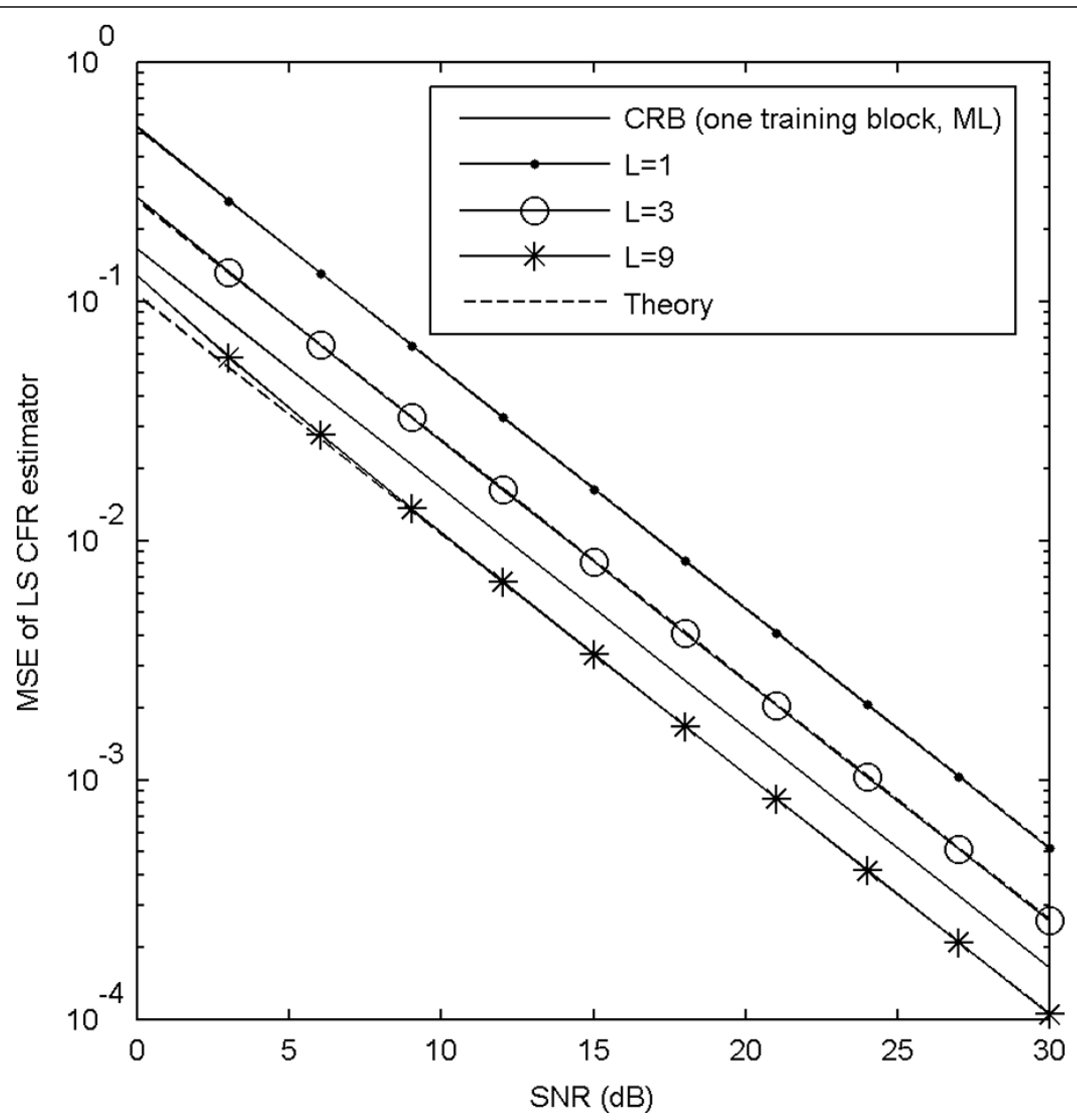

Figure 2 Performance of LS channel estimator after LS frequency offset correction. With initial frequency offset $\delta=0.2$ and for $L=1,3,9$, shown here are MSE of LS CFR estimator versus SNR curves by Monte Carlo simulations as well as by theory. The CRB curve is for ML CFR estimator using one OFDM training block.

Next, we would like to examine the impact of our estimators on the system error rate performance. We shall take square 16-QAM signaling for the OFDM system over frequency-selective Rayleigh fading channels. As before, we again assume a channel length of $v=9$ and an OFDM block length of $N=64$ samples. Also, we choose an exponential channel power profile with normalized channel power. Define the received SNR for $k$ th subcarrier as $\gamma_{k}=\left|H_{k}\right|^{2} \sigma_{X}^{2} / \sigma_{W}^{2}$, where $\sigma_{X}^{2}=E\left[\left|X_{k}\right|^{2}\right]$ is the average transmitted data power, and the total average SNR as $\bar{\gamma}=\frac{1}{N} \sum_{k=0}^{N-1} \bar{\gamma}_{k}$ with $\bar{\gamma}_{k}=E\left[\left|H_{k}\right|^{2}\right] \sigma_{X}^{2} / \sigma_{W}^{2}$. It can readily be shown that $\bar{\gamma}=E[\gamma]$, where $\gamma=\left\|\tilde{\mathbf{y}}_{0}\right\|^{2} / N \sigma_{w}^{2}$ is just the block SNR defined earlier in Section 5. Then, for initial frequency offset of $\delta=0.2$, Figure 7 presents the Monte Carlo simulation results of SER versus average SNR $\bar{\gamma}$ for the OFDM system employing LS frequency tracking combined with channel estimation with $L=1$ and $L=3$. Both LS and ML channel estimations are tested. Also included in Figure 7 for comparison is the ideal system 16-QAM SER with perfect frequency synchronization and channel estimation. In fact the ideal QAM SER for an OFDM system over a Rayleigh fading channel can readily be derived in a closed form by using the moment generating function approach given in [9]. We shall not carry out the derivation here, which will carry us afar and take too much space but present the final result as follows:

$$
P_{M}=\frac{1}{M}\left[M-1-\frac{2 a(\sqrt{M}-1)}{\sqrt{1+a^{2}}}-\frac{4 a(\sqrt{M}-1)^{2}}{\pi \sqrt{1+a^{2}}} \tan ^{-1} \frac{a}{\sqrt{1+a^{2}}}\right],
$$

where $M=16$ has been used for our square 16-QAM signaling and $a=\sqrt{3 \bar{\gamma} / 2(M-1)}$. From the figure, we can see that the Monte Carlo results for LS channel estimations are slightly above the ideal SER result with 


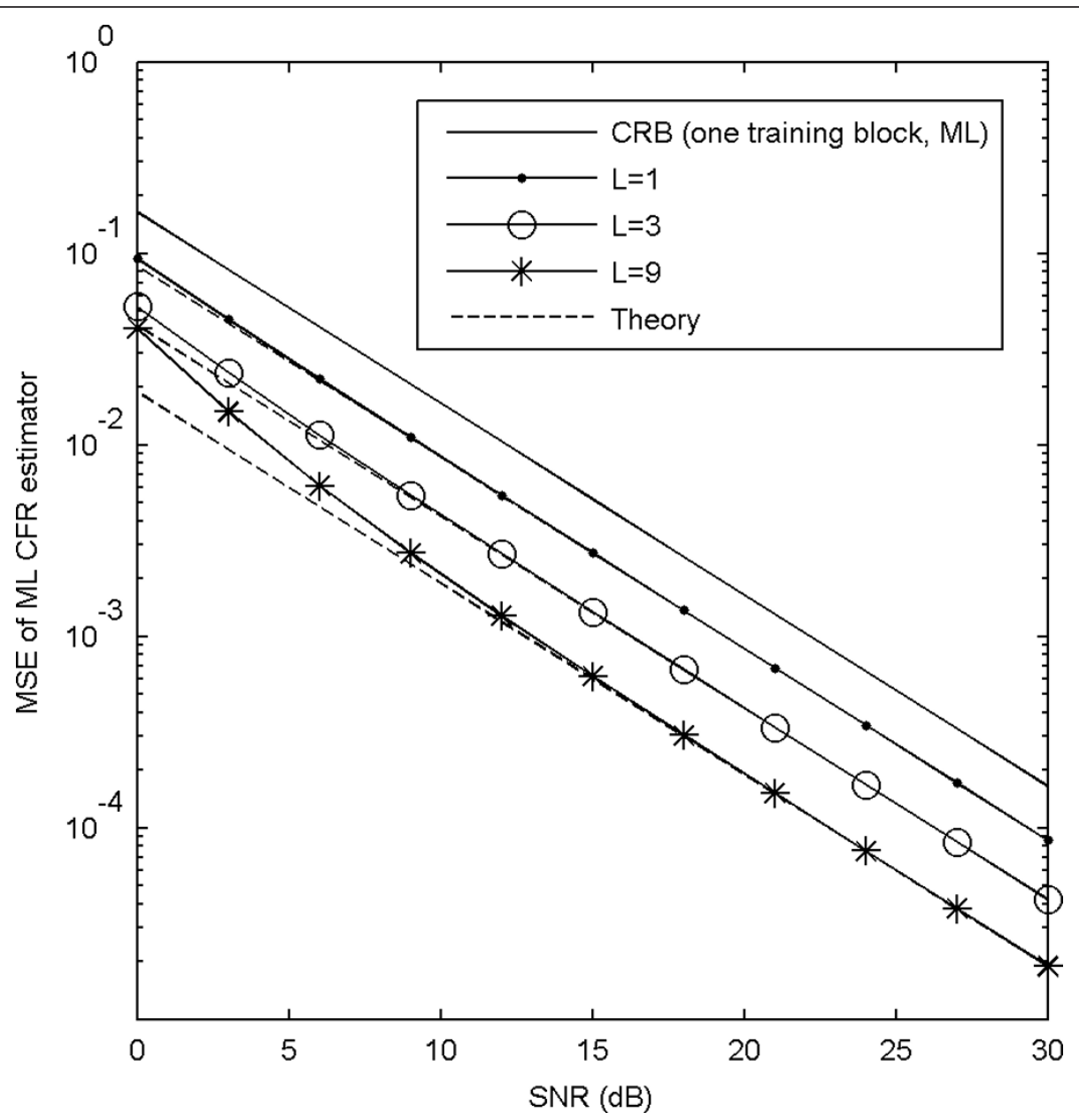

Figure 3 Performance of $\mathbf{M L}$ channel estimator after LS frequency offset correction. With initial frequency offset $\delta=0.2$ and for $L=1,3,9$, shown here are MSE of ML CFR estimator versus SNR curves by Monte Carlo simulations as well as by theory. The CRB curve is for ML CFR estimator using one OFDM training block.

the $L=3$ curve being better as expected, while the Monte Carlo curves for the ML channel estimations almost coincide with the ideal SER curve as the differences are not easily detected by eye. This further confirms the fact that using ML channel estimation after frequency tracking is better than LS channel estimation. We have performed similar simulations for various other values of $\delta$ including values close to $\delta=0.5$ and found the results very much the same as given by Figure 7. This means, in so far as the SER performance is concerned, within the tracking range of half the subcarrier spacing, the estimators of our algorithm are robust against the frequency offset.

Finally, Figure 8 compares SER performances versus SNR between various algorithms under $\delta=0.2$ using QAM transmission over the same channel as given for Figure 7. To compare with the LMMSE algorithm of [1], we have used Version B which has a faster convergence speed and wider estimation range than Version A.
In the comparisons, we take $L=1$ for our algorithms, $\mathrm{i}$. e., two repeated OFDM blocks are used for training. Thus, to be fair, we have also used two OFDM blocks as training data for the LMMSE [1] and the EM [5] algorithms so that same bandwidth efficiency is given for all algorithms under consideration. The simulation results show that our proposed algorithm using LS frequency tracking with ML channel estimator gives almost the same SER performance as those given by LMMSE and by the ideal case (i.e., perfect frequency tracking and channel estimation). While our algorithm using LS frequency tracking with LS channel estimation gives a slightly less SER performance. The EM algorithm given in [5] yields a very poor performance under $\delta=0.2$. Simulation results (not given here) show that the EM algorithm can only work when $\delta$ is very small (less than 0.02).

We note that the LMMSE has comparatively the best performance due to the additional iteration process 


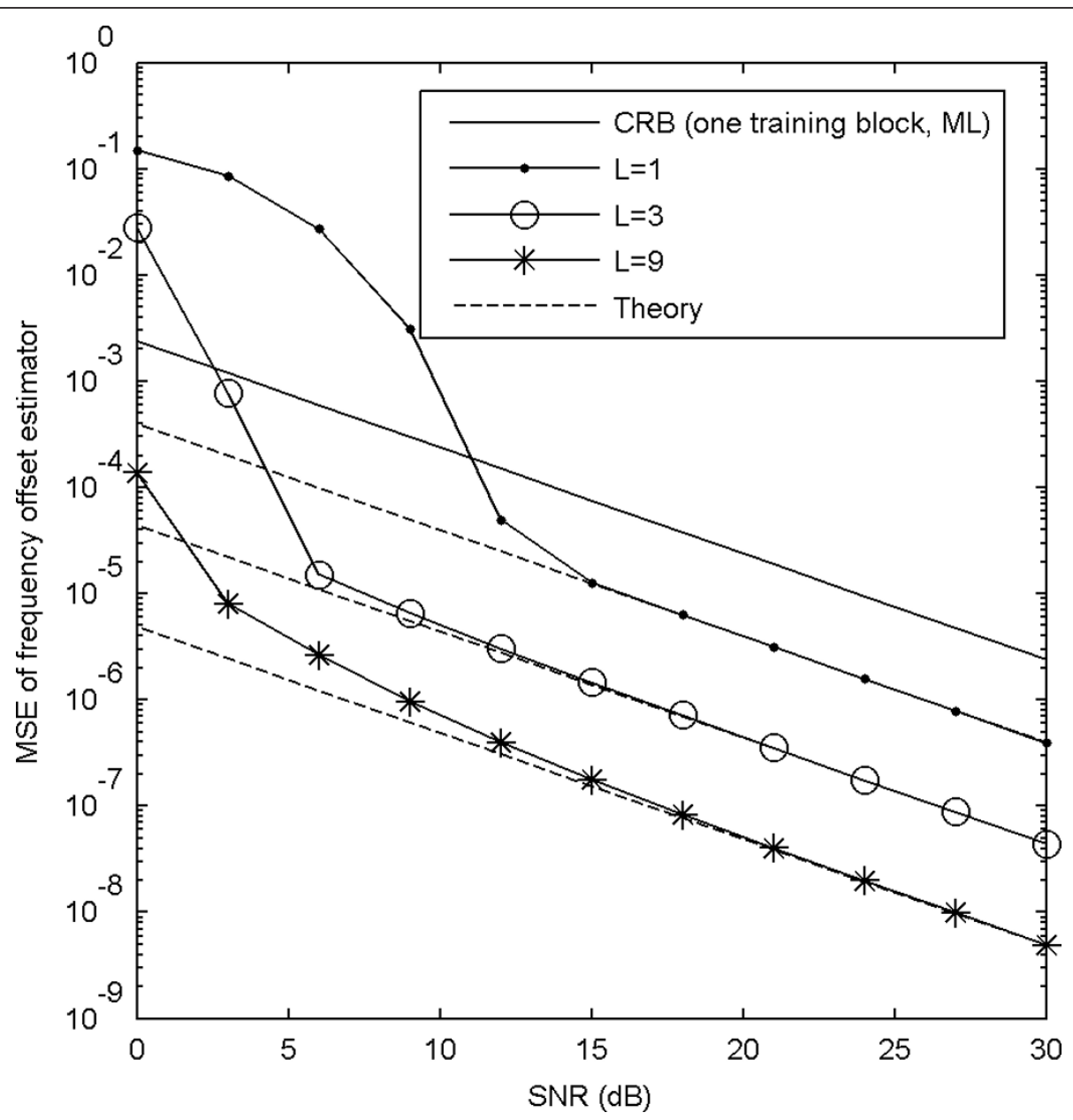

Figure 4 Performance of frequency tracking by method of least squares using $L+1$ OFDM training blocks. With initial frequency offset $\delta$ $=0.48$ and for $L=1,3,9$, shown here are MSE of frequency offset estimator versus SNR curves by Monte Carlo simulations as well as by theory. The CRB curve is for ML frequency estimator using one OFDM training block.

which gives further refined results. Nonetheless, our algorithm using LS frequency tracking with ML channel estimation yields a comparable performance but without the iteration process. This saves considerable computation time and hence contributes the major advantage of our algorithm. Table 1 displays the computational complexity needed for various algorithms. The complexity is counted by the arithmetic operations of two real numbers. One complex multiplication is equivalent to four real multiplications. Also, some composite data matrices can be calculated in advanced and stored in memory before performing estimation algorithm. $\frac{1}{N \sqrt{N}} \mathbf{F}_{L}^{H} \mathbf{X}^{H} \mathbf{F}_{N}$ in (17) of [1], for example, can be pre-computed and stored in memory for later use in adaptive iterations. The needed arithmetic operations displayed in Table 1 exclude such situations. Moreover, $S$ represents the number of iterative adaption needed in the proposed LMMSE algorithm [1]. Referring to the simulation results in [1], the number of iterations for achieving convergence for various values of CFOs is somewhere between 10 and 20. The reasonable value $S$ for use in practice is thus a little more than 20 .

As an example, we take $S=20$ and $v=N / 4$. Then, the LMMSE requires $40 N^{2}+240 N$ real multiplications, while the proposed LS frequency tracking with LS channel estimation needs $N^{2}+L N+1$ real multiplications, and the proposed LS frequency tracking with ML channel estimation takes only $\frac{N^{2}}{2}+L N+1$ real multiplications. Notably, the proposed LS frequency tracking with ML channel estimation has the least complexity, while the LMMSE algorithm has the heaviest load of mathematical operations.

\section{Conclusion}

By using repeated OFDM training blocks, we successfully implement the method of LS to perform frequency 


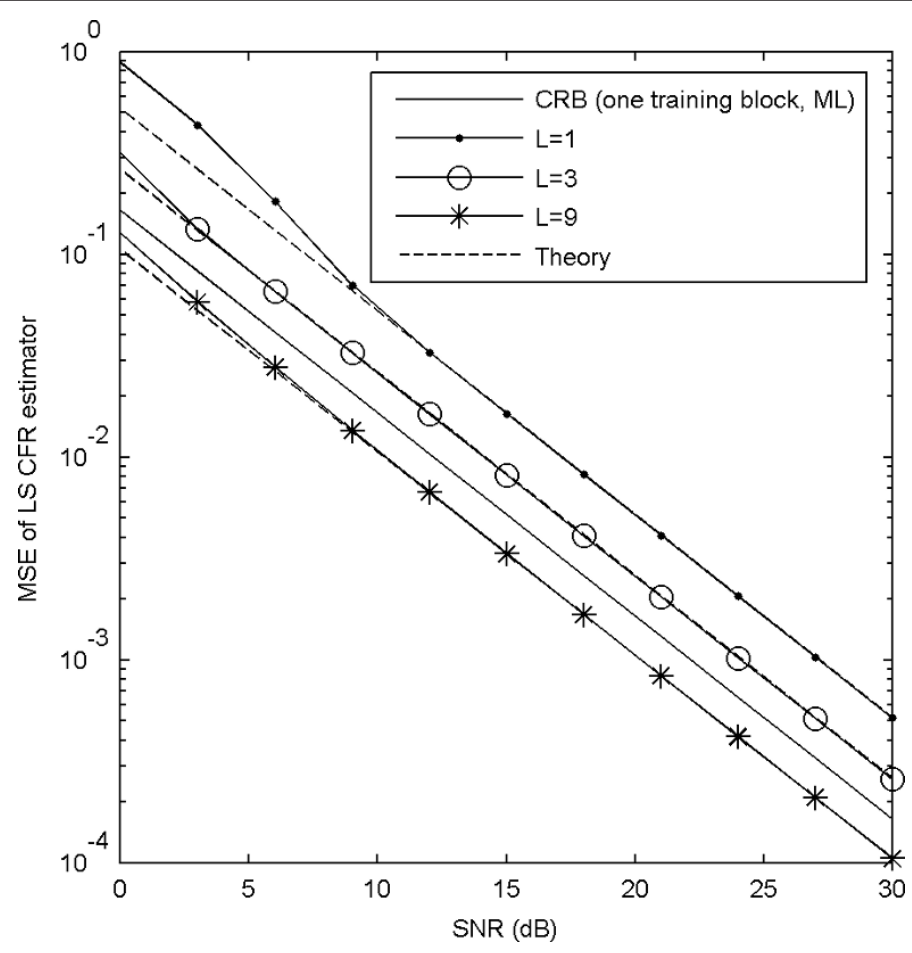

Figure 5 Performance of LS channel estimator after LS frequency offset correction. With initial frequency offset $\delta=0.48$ and for $L=1,3,9$, shown here are MSE of LS CFR estimator versus SNR curves by Monte Carlo simulations as well as by theory. The CRB curve is for ML CFR estimator using one OFDM training block.

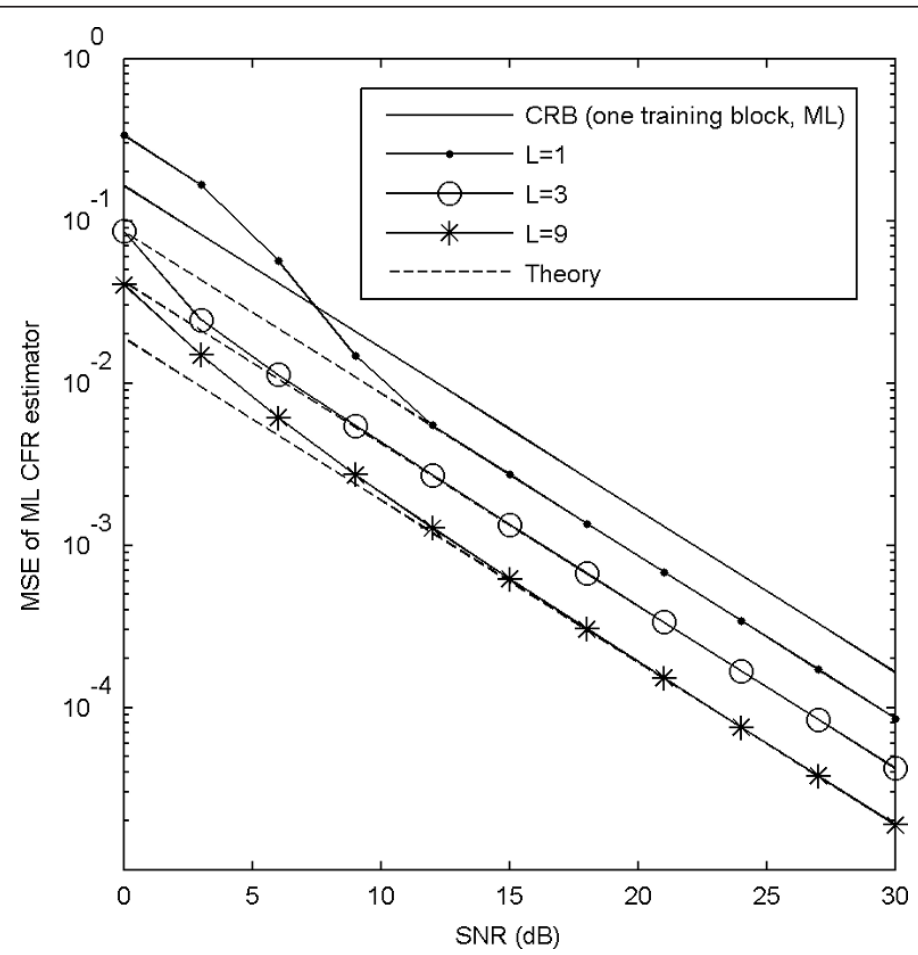

Figure 6 Performance of ML channel estimator after LS frequency offset correction. With initial frequency offset $\delta=0.48$ and for $L=1,3,9$, shown here are MSE of ML CFR estimator versus SNR curves by Monte Carlo simulations as well as by theory. The CRB curve is for ML CFR estimator using one OFDM training block. 


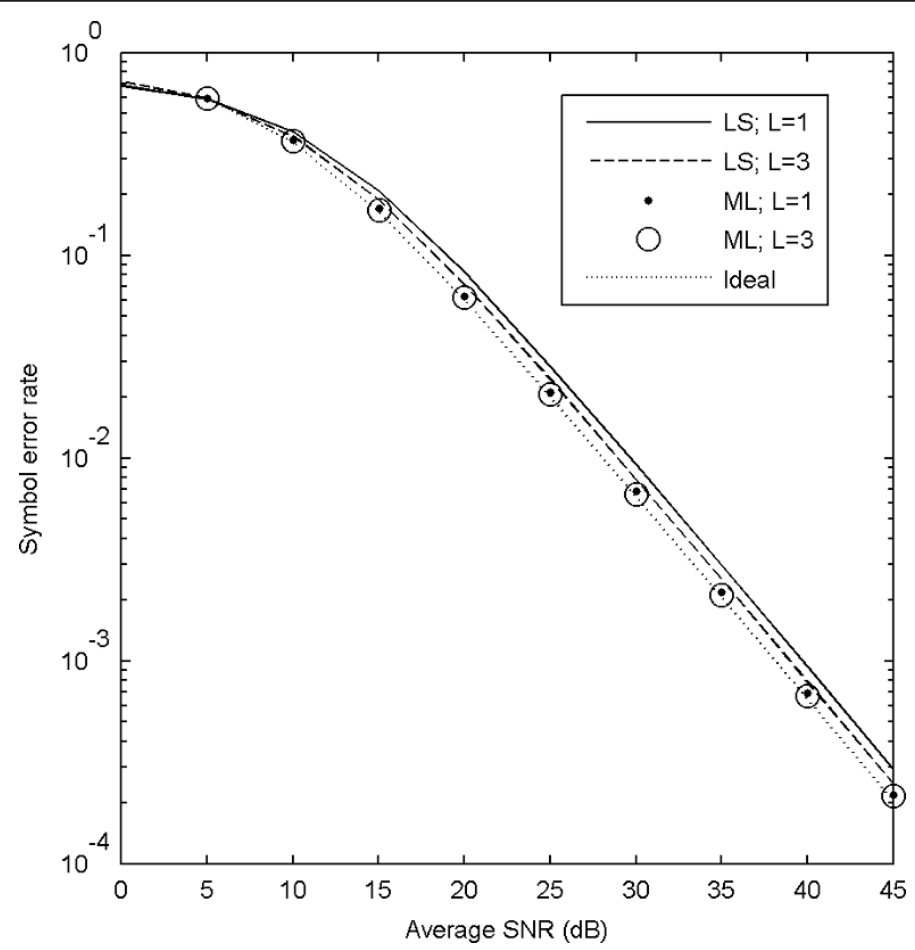

Figure 7 16-QAM SER versus total average SNR $\bar{\gamma}$ for OFDM in frequency-selective Rayleigh fading channels. The OFDM system employs LS frequency tracking combined with LS (ML) channel estimation for $\delta=0.2$. Also included for comparison is the ideal system 16-QAM SER with perfect frequency synchronization and channel estimation.

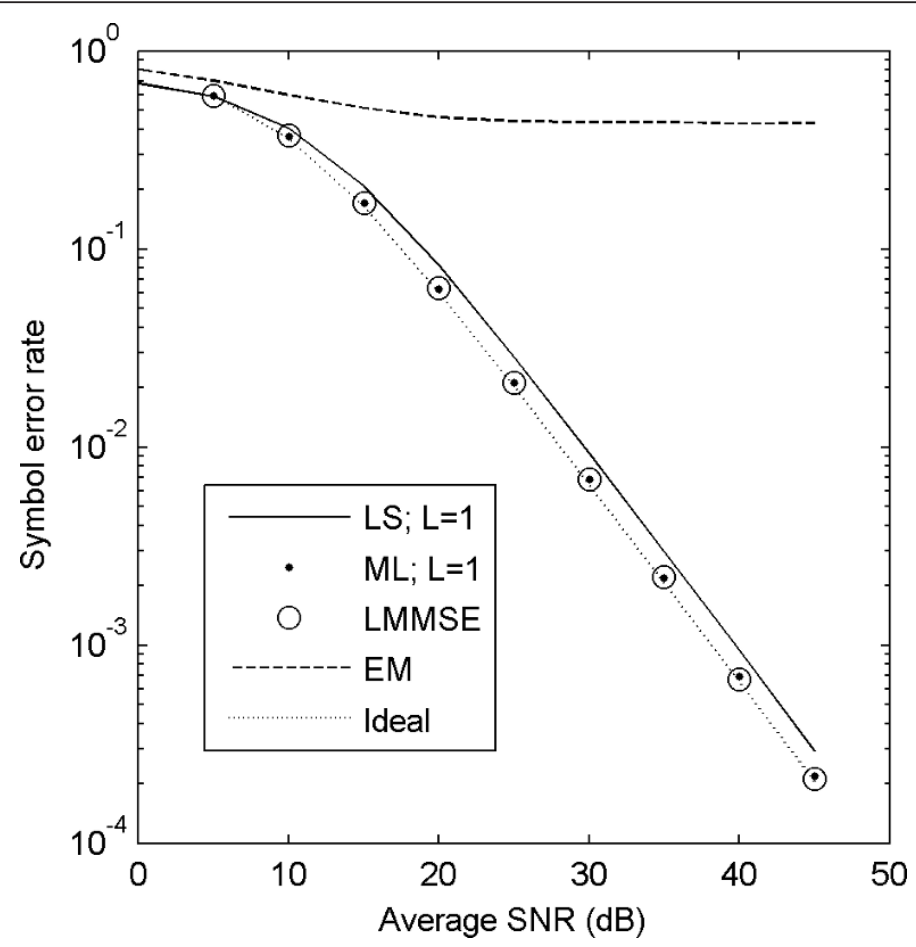

Figure 8 16-QAM SER versus total average SNR $\bar{\gamma}$ for various algorithms. The OFDM system employs two consecutive identical symbol blocks for frequency tracking and channel estimation for the same bandwidth efficiency. $\delta=0.2$. Also included is the ideal system 16-QAM SER with perfect frequency synchronization and channel estimation. 
Table 1 Computation complexity

\begin{tabular}{|c|c|c|c|}
\hline & LMMSE [1] & $\begin{array}{l}\text { The proposed algorithm with LS channel } \\
\text { estimation }\end{array}$ & $\begin{array}{l}\text { The proposed algorithm with ML channel } \\
\text { estimation }\end{array}$ \\
\hline Real multiplication & $\begin{array}{l}S(8 v+12) \\
N\end{array}$ & $L N+1+N^{2}$ & $L N+1+2 V N$ \\
\hline Real division & $S N$ & 1 & 1 \\
\hline $\begin{array}{l}\text { Arctangent } \\
\text { operation }\end{array}$ & $S N$ & 1 & 1 \\
\hline
\end{tabular}

tracking combined with channel estimation for OFDM systems over mobile wireless channels. Unlike the usual ML algorithms commonly adopted for joint frequency tracking and channel estimation using one OFDM training block, our algorithm has no local extrema problem arising from the highly nonlinear nature of the multidimensional log-likelihood function and does not require the use of time-consuming adaptive iterations. Simulation results show that both the tracking performance and the error rate performance for OFDM systems using our proposed method are very satisfactory.

\section{Abbreviations}

CFR: channel frequency response; CIR: channel impulse response; CRB: Cramer-Rao bound; DFT: discrete Fourier transform; i.i.d.: independent: identically distributed; LMMSE: linear minimum-mean-square-error; LS: least squares; ML: maximum-likelihood; MSE: mean square error; OFDM: orthogonal frequency division multiplexing; RV: random variable; SNR: signalto-noise ratio.

\section{Author details}

'Department of Electrical Engineering, Tamkang University, No. 151, Yingzhuan Rd., Tamsui Dist., New Taipei City 25137, Taiwan ${ }^{2}$ Department of Electrical Engineering, Fu Jen Catholic University, No. 510, Zhongzheng Rd. Xinzhuang Dist., New Taipei City 24205, Taiwan ${ }^{3}$ Department of Computer Science and Engineering, Tatung University, No. 40, Sec. 3, Zhongshan N. Rd., Taipei City 104, Taiwan

\section{Competing interests}

The authors declare that they have no competing interests.

Received: 5 December 2011 Accepted: 8 June 2012

Published: 8 June 2012

\section{References}

1. HY Liu, RY Yen, Effective adaptive iteration algorithm for frequency tracking and channel estimation in OFDM systems. IEEE Trans Veh Technol. 59(4), 2093-2097 (2010)

2. M Morelli, U Mengali, Carrier-frequency estimation for transmissions over selective channels. IEEE Trans Commun. 48(9), 1580-1589 (2000). doi:10.1109/26.870025

3. X Ma, H Kobayashi, SC Schwartz, Joint frequency offset and channel estimation for OFDM, in Proceedings of the IEEE GLOBECOM 2003, San Francisco, USA, 1, 15-19 (December 2003)

4. L Zheng, W Cheng, J Hu, D Yuan, The ML frequency tracking algorithm for OFDM systems based on pilot symbols and decision data. in Proceedings of the IEEE Symposium on Microwave, Antenna, Propagation, and EMC Technology for Wireless Communication. Beijing, China. 2, 1611-1614 (August 2005)

5. JH Lee, JC Han, SC Kim, Joint carrier frequency synchronization and channel estimation for OFDM systems via the EM algorithm. IEEE Trans Veh Technol. 55(1), 167-172 (2006). doi:10.1109/TVT.2005.861212
6. FZ Merli, GM Vitetta, Iterative ML-based estimation of carrier frequency offset, channel impulse response and data in OFDM transmissions. IEEE Trans Commun. 56(3), 497-506 (2008)

7. P Stoica, $O$ Besson, Training sequence design for frequency offset and frequency-selective channel estimation. IEEE Trans Commun. 51(11), 1910-1917 (2003). doi:10.1109/TCOMM.2003.819199

8. DC Chu, Polyphase codes with good periodic correlation properties. IEEE Trans Inf Theory. 18(4), 531-532 (1972). doi:10.1109/TIT.1972.1054840

9. MS Alouin, A Goldsmith, A unified approach for calculating error rates of linearly modulated signals over generalized fading channels. IEEE Trans Commun. 47(9), 1324-1334 (1999). doi:10.1109/26.789668

doi:10.1186/1687-1499-2012-192

Cite this article as: Yen et al:: Frequency tracking by method of least squares combined with channel estimation for OFDM over mobile wireless channels. EURASIP Journal on Wireless Communications and Networking 2012 2012:192.

\section{Submit your manuscript to a SpringerOpen ${ }^{\mathcal{O}}$ journal and benefit from:}

- Convenient online submission

- Rigorous peer review

- Immediate publication on acceptance

- Open access: articles freely available online

- High visibility within the field

- Retaining the copyright to your article

Submit your next manuscript at $>$ springeropen.com 